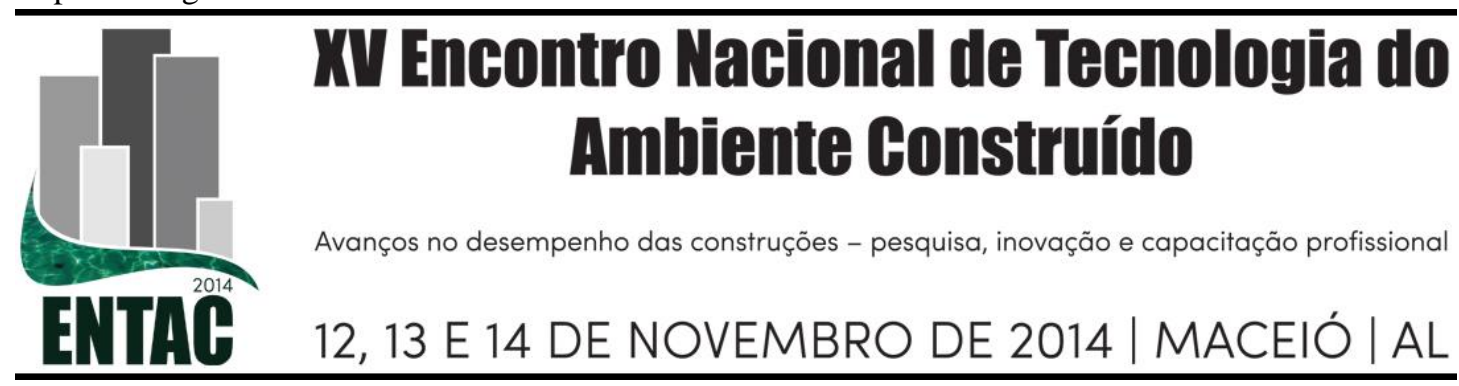

\title{
ANÁLISE DA PRODUÇÃO CIENTÍFICA RELACIONADA AO CUSTEIO-META (TARGET COSTING) NA CONSTRUÇÃO CIVIL NOS ÚLTIMOS 5 ANOS (2009-2013)
}

\author{
CÂNDIDO, Luis Felipe (1); BARRETO, José Maurício Lima (2); BARROS
}

NETO, José de Paula (3)

(1) PEC/UFC, e-mail: luisf_civil@yahoo.com.br, (2) PEC/ UFC, e-mail: mauriciobarreto@ufc.br, (3) GERCON/UFC, e-mail: barrosneto@gercon.ufc.br

\begin{abstract}
RESUMO
O custeio-meta (ou target costing) é uma estratégia eficaz na busca do equilíbrio entre o custo x preço, qualidade e funcionalidade. Desenvolvida na manufatura, a aplicação destes conceitos na construção civil vem aumentando e adquirindo maturidade. Diante do exposto, a presente pesquisa é um estudo bibliométrico cujo objetivo principal é descrever o quadro de publicações sobre custeio-meta na construção civil nos últimos 5 anos (2009-2013). Como resultado, foi observado um total de 30 publicações com parcela significativa de contribuição nacional (16 publicações ou $53 \%$ da amostra). Verificou-se também que as principais fontes de publicações foram a conferência anual da comunidade da construção enxuta (IGLC, com 36\%) e os encontros da ANTAC (ENTAC e SIBRAGEC, com 17\%). Os temas mais recorrentes foram custeio-meta no contexto de habitações de interesse social, gerenciamento de projeto, processo de projeto e desenvolvimento de novos produtos. Por fim, pode-se destacar a contribuição de pesquisas qualitativas $(33 \%)$ e $83 \%$ de estudos empíricos, o que remete a afirmativa inicial de que a aplicação do custeio-meta na construção civil está em processo de maturação. Como contribuição, espera-se atualizar e expandir os estudos que já foram desenvolvidos no tema consolidando o eixo referencial do conhecimento.
\end{abstract}

Palavras-chave: construção civil, gestão de custos, custeio-meta, bibliometria.

\begin{abstract}
The target costing approach can be characterized as effective strategy to equalize cost $x$ price, quality and functionality. Developed in manufacturing industry, the application of these concepts in construction industry has growth and acquiring maturity. Then, this paper aims to analyze the scientific publications about the target costing in building construction in the last 5 years (2009-2013) in a bibliometric approach. As result, 36 papers were found with a significant participation of Brazil (16 papers or 53\% of the sample). The principal sources of publications observed were the annual IGLC conference (36\%) and events of ANTAC (ENTAC e SIBRAGEC, with 17\%). The most frequently themes were target costing in context of social housing, project management, project process and development of new products. Finally, the qualitative research was used in 33\% of sample, while $83 \%$ from this sample was empirical study. This information reinforces the maturity process which the target costing has been improving. With this paper, authors hope provide a vision about this theme in the building industry, consolidating and spreading it like a knowledge area.
\end{abstract}

Keywords: building construction, cost management, target costing, bibliometric analysis.

\section{INTRODUÇÃ̃O}

A comunicação científica tem um papel importante na transmissão do conhecimento tendo em vista o tripé ensino-pesquisa-extensão, no sentido de geração do 
conhecimento, manutenção, desenvolvimento de novas tecnologias, aplicação e aceitação do que é produzido cientificamente como constituinte do conhecimento científico (OLIVEIRA, 2002).

Para comunicação científica tornar-se efetiva, uma das maneiras mais utilizadas e a mais importante é a publicação de trabalhos científicos em periódicos acadêmicos (RIBEIRO et al., 2007).

Devido a uma forte profusão de trabalhos resultante do surgimento dos periódicos eletrônicos os pesquisadores, muitas vezes, encontram a dificuldade para selecionar fontes bibliográficas de pesquisa dada a quantidade de trabalhos que estão sendo publicados, principalmente após a criação dos periódicos eletrônicos (FAPESP, 2011).

Uma forma de pesquisa que visa clarificar e simplificar o acesso de pesquisadores aos principais artigos de suas respectivas áreas é o artigo bibliométrico (ou estudo de produção científica sobre um determinado assunto) (PITHAN et al., 2005).

Este tipo de trabalho busca entender, mapear, mensurar as referências bibliográficas e as suas publicações, conhecer o impacto de determinados autores ou periódicos relacionados a correntes de pensamento e/ou a produção científica de uma determinada área/tema proporcionando a formação de referencial teórico para futuras pesquisas na área (CARDOSO et al., 2005 apud ROCHA et al., 2010; CAMPOS et al., 2012)

Ao se revisitar a literatura pertinente, verificou-se uma carência de estudos bibliométricos específicos sobre target costing na construção civil, tornando o presente trabalho pertinente e justificável mediante aos resultados oferecidos pela bibliometria.

Diante do exposto, a presente pesquisa é um estudo bibliométrico cujo objetivo principal é descrever o quadro de publicações sobre custeio-meta na construção civil nos últimos 5 anos (2009-2013).

Como contribuição espera-se atualizar e expandir os estudos que já foram desenvolvidos no tema consolidando o eixo referencial do conhecimento da temática através do estudo bibliométrico.

\section{CUSTEIO-META (TARGET COSTING)}

O custeio-meta (ou target costing) é uma estratégia eficaz na busca do equilíbrio entre o tripé custo x preço, qualidade e funcionalidade, elementos que constituem o conceito de sobrevivência da empresa no mercado. (COOPER; SLAGMULDER, 1997 apud GRANJA et al., 2011).

A diferença principal do custo-meta para outras ferramentas que visam à redução de custos está no estabelecimento de metas de custos a serem atingidos com enfoque na fase de desenvolvimento de produtos (KERN, 2005).

Normalmente a temática de custeio-meta está ligada a de engenharia de valor e ao custeio kaizen. A engenharia de valor é definida como esforços organizados no sentido de implantar análise funcional de produtos e serviços para atingir, com confiabilidade, todas as funções requeridas ao menor custo de ciclo de vida possível. Já o custeio kaizen consiste na redução de custos durante a etapa de produção e tem como objetivo principal buscar reduções de custo em todas as etapas da manufatura para ajudar a eliminar qualquer diferença entre o lucro-alvo e o lucro estimado (GRANJA et al., 2011) 
Desta forma, a utilização conjunta do custo-meta, engenharia de valor e custeio kaizen tem por objetivo estabelecer o custo com que um produto deve ser produzido, tendo em vista o preço pré-fixado pelo mercado, assegurando um nível satisfatório de desempenho e qualidade.

\section{METODOLOGIA}

\subsection{Enquadramento metodológico}

Quanto à natureza dos dados, a presente pesquisa enquadra-se como quantitativa com objetivos exploratórios e descritivos. (COLLIS; HUSSEY, 2005). Exploratório devido à incipiência de trabalhos bibliométricos, buscando dessa forma conhecer, identificar e levantar os trabalhos no tema em apreço. Descritivo, pois visa caracterizar, mapear e descrever como estas publicações vêm sendo conduzidas (RICHARDSON, 2011).

Em relação à lógica aplicada, caracteriza-se como dedutiva, pois as discussões produzidas são fruto de uma amostra representativa da realidade de publicações na área (COLLIS; HUSSEY, 2005).

Quanto aos resultados classifica-se como básica, pois tem função de sistematizar o conhecimento buscando categorizar as principais publicações, autores e instituições, metodologias em uso e fontes bibliográficas que alimentam o arcabouço teórico, sem, no entanto, se aprofundar em uma questão específica dentro da temática de custeiometa. (COLLIS; HUSSEY, 2005).

A coleta dos dados deu-se essencialmente por pesquisa bibliográfica através de um levantamento sistemático a partir de anais de congresso e periódicos eletrônicos da área.

\subsection{Delineamento da pesquisa}

A Figura 1 apresenta o delineamento da pesquisa cujas etapas foram detalhadas a seguir.

Figura 1 - Delineamento da pesquisa

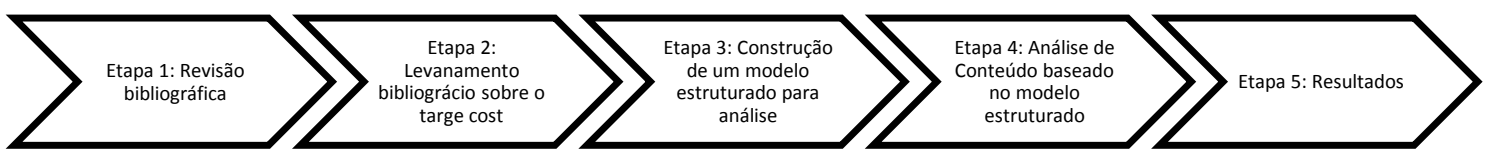

Fonte: dos autores.

Para o levantamento da bibliografia, utilizou-se o Portal da CAPES com uma busca através das palavras-chave: "target cost*"; custeio-meta; custo meta; custeio kaizen. Delimitou-se a pesquisa por assunto e em um horizonte de 5 anos (2009 - 2013). O horizonte de 5 anos foi escolhido devido o cálculo de Fatores de Impacto como Journal Citation Report $^{\circledR}$ da Thomson Reuters utilizarem está margem de tempo.

A pesquisa ao portal da CAPES resultou em 33 trabalhos dos quais após uma filtragem inicial resultou em uma amostra de 21 trabalhos. As referências bibliográficas destes artigos foram exploradas o que aumentou a amostra para 41 trabalhos. Destes 41 trabalhos, 5 foram descartados por não tratarem especificamente do tema voltado a construção ou suas linhas de pesquisa. Assim, a amostra final ficou constituída de 36 trabalhos. 


\subsection{Protocolo de análise}

Baseado nos trabalhos de Pithan et al. (2005), Rocha et al. (2010), Campos et al. (2012) e Lacerda et al. (2012) foi produzido um protocolo de análise que foi subdividido em:

- Identificação dos artigos: ano de publicação, tipo, título, evento/ periódico, tema;

- Autores e instituições: autores, titulação acadêmica, vínculo institucional do autor, $n^{\circ}$ de autores, ponderação dos autores, instituições, origem da instituição;

- Metodologia: existência ou não de estudo empírico, detalhamento ou não do método de pesquisa, natureza da pesquisa e estratégia de pesquisa;

- Citações (referencial bibliográfico): autores, cronologia, tipo de bibliografia, fontes das citações e referências bibliográficas.

\section{RESULTADOS}

\subsection{Identificação dos artigos}

A amostra final resultou em 36 trabalhos nos mais diversos temas e tipos. Por questões de escopo, a listagem destes artigos não foi apresentada. De maneira geral, os trabalhos distribuíram-se cronologicamente conforme a Tabela 1.

Tabela 1 - Comportamento das publicações ao longo dos anos analisa

\begin{tabular}{lcccccc}
\hline \multicolumn{1}{c}{ Ano } & $\mathbf{2 0 0 9}$ & $\mathbf{2 0 1 0}$ & $\mathbf{2 0 1 1}$ & $\mathbf{2 0 1 2}$ & $\mathbf{2 0 1 3}$ & Total \\
\hline Número de trabalhos & 6 & 5 & 8 & 8 & 3 & 30 \\
Número de Autores & 8 & 9 & 15 & 15 & 5 & 52 \\
Número de Universidades & 5 & 6 & 5 & 7 & 4 & 27 \\
\hline
\end{tabular}

Fonte: dos autores.

Em relação às fontes de publicação há uma evidente polarização entre artigos de congresso $(63,33 \%)$ e artigos de revista $(26,67 \%)$. Também compõe o quadro $6,67 \%$ (dissertação de mestrado) e 3,33\% (outros).

Liderando o ranking de fontes de publicação encontra-se a conferência do International Group for Lean Construction (IGLC) com 11 publicações, conforme a Tabela 2.

Tabela 2 - Fonte das publicações sobre custeio-meta

\begin{tabular}{cccc}
\hline $\mathbf{N}^{\mathbf{2}}$ & Fonte & Total & \% de Trabalhos \\
\hline 1 & IGLC & 11 & $36,67 \%$ \\
2 & Revista Ambiente Construído & 3 & $10,00 \%$ \\
3 & ENTAC & 3 & $10,00 \%$ \\
4 & SIBRAGEC & 2 & $6,67 \%$ \\
5 & Construction Research Congress & 2 & $6,67 \%$ \\
6 & Unicamp (Dissertações de Mestrado) & 2 & $6,67 \%$ \\
7 & Jounal of Lean Construction & 1 & $6,67 \%$ \\
8 & Architetural Engineering and Design Management & 1 & $3,33 \%$ \\
9 & Construction Mangament and Economics & $3,33 \%$
\end{tabular}


Tabela 2 - Fonte das publicações sobre custeio-meta

\begin{tabular}{cccc}
\hline $\mathbf{N}^{\mathbf{0}}$ & Fonte & Total & \% de Trabalhos \\
\hline 10 & ELECS & 1 & $3,33 \%$ \\
11 & Journal of Financial Management of Property and Construction & 1 & $3,33 \%$ \\
12 & VTT Tiedotteita, Espoo (Relatório Técnico) & 1 & $3,33 \%$ \\
\hline
\end{tabular}

Fonte: dos autores.

Após uma discussão prévia entre os autores, os artigos foram classificados em 5 categorias cuja descrição é apresentada a seguir e a distribuição percentual e cronológica são apresentadas na Tabela 3.

Categoria 1 (Est. Custos) - agrupa trabalhos com objetivo de modelar os custos, elaborar plano de custos e realizar estimativas de custos. Categoria 2 (Des. Produtos) agrupa trabalhos sobre processo de desenvolvimento do produto na construção. Categoria 3 (Proc. de Proj.) - agrupa trabalhos que dissertam sobre o processo de projeto. Categoria 4 (HIS) - agrupa trabalhos sobre aplicações do target costing em habitações de interesse social. Categoria 5 (Map. Lit.) - agrupa trabalhos que buscam traçar de maneira geral o eixo teórico referente a temáticas ligadas ao target costing (como mapeamento sistemático da literatura, por exemplo).

Tabela 3 - Classificação por tema em ordem cronológica

\begin{tabular}{|c|c|c|c|c|c|c|c|c|c|c|c|c|}
\hline Tema & & 2009 & & 2010 & & 2011 & & 2012 & & 2013 & & otal \\
\hline (1) Est. Custos & 3 & $50 \%$ & 0 & $0 \%$ & 3 & $38 \%$ & 1 & $17 \%$ & 0 & $0 \%$ & 7 & $23 \%$ \\
\hline (2) Des. Produtos & 1 & $17 \%$ & 1 & $14 \%$ & 2 & $25 \%$ & & $0 \%$ & 2 & $67 \%$ & 6 & $20 \%$ \\
\hline (3) Proc. de Proj. & 0 & $0 \%$ & 3 & $43 \%$ & 2 & $25 \%$ & 3 & $50 \%$ & 0 & $0 \%$ & 8 & $27 \%$ \\
\hline (4) HIS & 1 & $17 \%$ & 2 & $29 \%$ & 1 & $13 \%$ & 2 & $33 \%$ & 0 & $0 \%$ & 6 & $20 \%$ \\
\hline (5) Map. Lit. & 1 & $17 \%$ & 1 & $14 \%$ & 0 & $0 \%$ & & $0 \%$ & 1 & $33 \%$ & 3 & $10 \%$ \\
\hline Total & 6 & $100 \%$ & 7 & $100 \%$ & 8 & $100 \%$ & 6 & $100 \%$ & 3 & $100 \%$ & 30 & $100 \%$ \\
\hline
\end{tabular}

Fonte: dos autores.

\subsection{Autores e instituições}

A Tabela 4 apresenta os 5 autores principais baseados na ponderação Lakmazaheri e Rasdorf (1998 apud Pithan et al., 2005) e no número de artigos publicados como autor principal e coautor.

Tabela 4 - 5 autores principais de 2009-2013

\begin{tabular}{cccccc}
\hline \multirow{2}{*}{$\mathbf{N}^{\mathbf{0}}$} & \multirow{2}{*}{ Autor } & \multirow{2}{*}{ Ponderação } & \multicolumn{2}{c}{ Artigos como } & \multirow{2}{*}{ Total } \\
\cline { 4 - 5 } & & & principal & coautor & \\
\hline 1 & BALLARD, G. & 5,08 & 4 & 6 & 10 \\
2 & GRANJA, A. D. & 4,42 & 1 & 10 & 11 \\
3 & JACOMIT, A. M. & 2,5 & 4 & 1 & 5 \\
4 & RUIZ, J. DE A. & 1,75 & 3 & 0 & 3 \\
5 & PENNANEN, A. & 1,67 & 2 & 2 & 4 \\
\hline
\end{tabular}

Fonte: dos autores. 
Quanto à titulação acadêmica destes autores, observa-se que 47\% dos autores são doutores e $\mathrm{PhD}$, seguidos de mestres (16\%), Graduados (5\%), não informados (25\%) e $7 \%$ de outros. Observa-se ainda que $45 \%$ dos autores são professores, seguidos de estudantes de mestrado (13\%), graduandos (6\%), doutorandos (4\%), não informado $(6 \%)$ e outros $(26 \%)$.

A Tabela 5 apresenta o ranking por ordem de colaboração.

Tabela 5 - Ranking de instituições colaboradoras

\begin{tabular}{ccccccccc}
\hline \multirow{2}{*}{ Instituição } & \multicolumn{7}{c}{ Participação em artigos } & \multirow{2}{*}{ Total } \\
\cline { 2 - 7 } & $\mathbf{2 0 0 9}$ & $\mathbf{2 0 1 0}$ & $\mathbf{2 0 1 1}$ & $\mathbf{2 0 1 2}$ & $\mathbf{2 0 1 3}$ & & \\
\hline Unicamp & 3 & 1 & 4 & 4 & 1 & 13 & $29,55 \%$ \\
University of California & 2 & 1 & 2 & 3 & 2 & 10 & $22,73 \%$ \\
Tampere University of Technology & 0 & 1 & 1 & 0 & 2 & 4 & $9,09 \%$ \\
Universidade Estadual de Londrina & 0 & 1 & 2 & 0 & 0 & 3 & $6,82 \%$ \\
\hline$\sum \mathbf{4}$ primeiros & $\mathbf{5}$ & $\mathbf{4}$ & $\mathbf{9}$ & $\mathbf{7}$ & $\mathbf{5}$ & $\mathbf{3 0}$ & $\mathbf{6 8 \%}$ \\
\hline$\sum$ demais & $\mathbf{3}$ & $\mathbf{4}$ & $\mathbf{1}$ & $\mathbf{5}$ & $\mathbf{1}$ & $\mathbf{1 4}$ & $\mathbf{3 2 \%}$ \\
\hline Total & $\mathbf{8}$ & $\mathbf{8}$ & $\mathbf{1 0}$ & $\mathbf{1 2}$ & $\mathbf{6}$ & $\mathbf{4 4}$ & $\mathbf{1 0 0 \%}$ \\
\hline
\end{tabular}

Fonte: dos autores.

Destaca-se a surpreendente colaboração da Universidade Estadual de Campinas (Unicamp) como instituição com maior colaboração de autores nas publicações examinadas e que, juntamente com a Universidade da Califórnia-Berkeley são as únicas instituições que tem publicações em todos os anos examinados (2009-2013)

Em relação à origem das instituições cujos autores têm vinculação, verifica-se que apenas 5 países fazem parte desta amostra com um total de 18 autores e 22 coautores conforme a Figura 2.

Figura 2 - Distribuição geográfica das instituições que publicaram no período

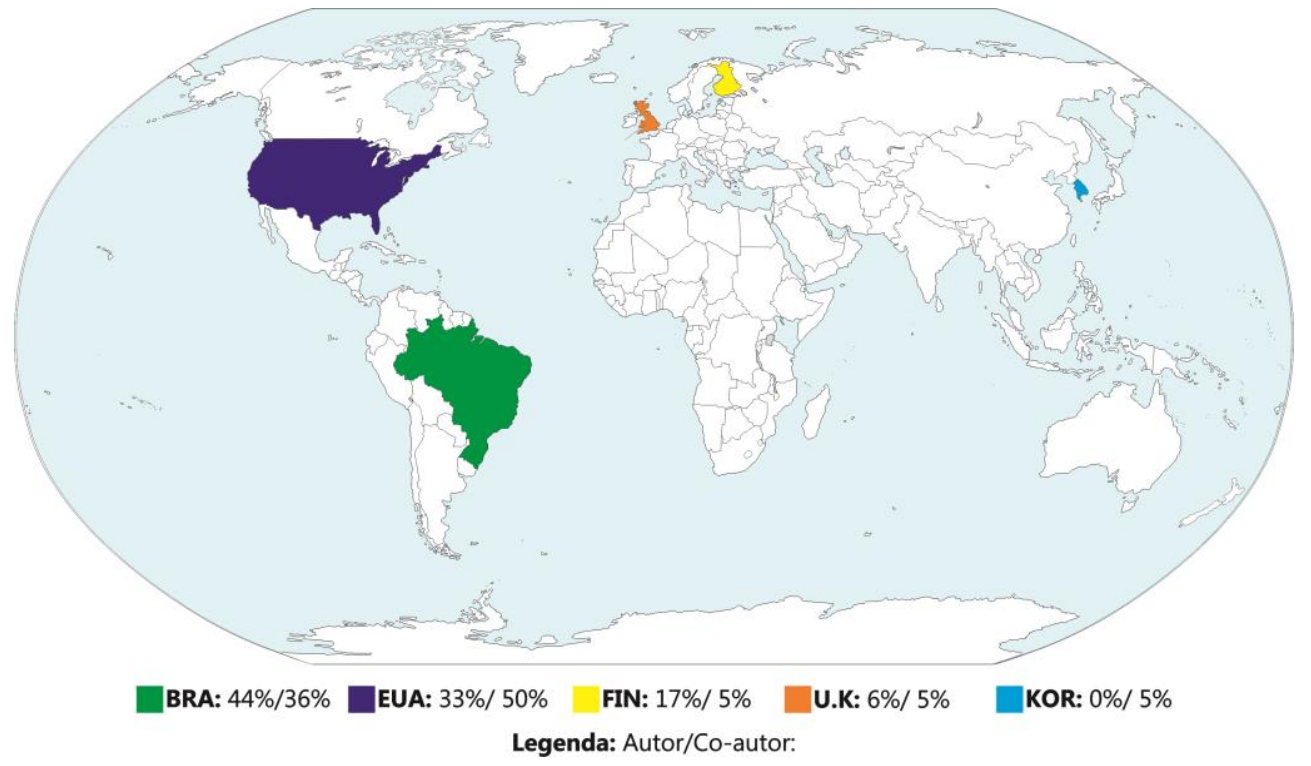

Fonte: dos autores. 


\subsection{Metodologia}

Observa-se que os trabalhos com estudo empírico predominam com $64 \%$ do total de trabalhos, destacando a estratégia de pesquisa estudo de caso. Este dado indica uma transição de estudos teóricos, embora ainda de forma incipiente, para estudos sobre aplicações desta estratégia na construção civil, conforme a

Tabela 6.

Tabela 6 - Quantidade de estudos empíricos e suas estratégias ao longo dos anos

\begin{tabular}{|c|c|c|c|c|c|c|c|c|c|c|c|c|}
\hline \multirow{2}{*}{$\begin{array}{c}\text { Estratégias de } \\
\text { pesquisa }\end{array}$} & \multicolumn{10}{|c|}{ Ano } & \multirow{2}{*}{\multicolumn{2}{|c|}{ Total }} \\
\hline & & 2009 & & 2010 & & 2011 & & 2012 & & 2013 & & \\
\hline Estudo de caso & 4 & $67 \%$ & 2 & $40 \%$ & 4 & $50 \%$ & 2 & $25 \%$ & 0 & $0 \%$ & 12 & $40 \%$ \\
\hline Não informada & 0 & $0 \%$ & 3 & $60 \%$ & 4 & $50 \%$ & 5 & $63 \%$ & 2 & $67 \%$ & 14 & $47 \%$ \\
\hline Pesquisa Ação & 1 & $17 \%$ & 0 & $0 \%$ & 0 & $0 \%$ & 1 & $13 \%$ & 0 & $0 \%$ & 2 & $7 \%$ \\
\hline Rev.Bibliográfica & 1 & $17 \%$ & 0 & $0 \%$ & 0 & $0 \%$ & 0 & $0 \%$ & 0 & $0 \%$ & 1 & $3 \%$ \\
\hline Survey & 0 & $0 \%$ & 0 & $0 \%$ & 0 & $0 \%$ & 0 & $0 \%$ & 0 & $0 \%$ & 0 & $0 \%$ \\
\hline Map. da Literatura & 0 & $0 \%$ & 0 & $0 \%$ & 0 & $0 \%$ & 0 & $0 \%$ & 1 & $33 \%$ & 1 & $3 \%$ \\
\hline Est. Empírico & 5 & $83 \%$ & 2 & $40 \%$ & 5 & $63 \%$ & 5 & $63 \%$ & 1 & $33 \%$ & 18 & $60 \%$ \\
\hline Est. não empíricos & 1 & $17 \%$ & 3 & $60 \%$ & 3 & $38 \%$ & 3 & $38 \%$ & 2 & $67 \%$ & 12 & $40 \%$ \\
\hline Total & 6 & $100 \%$ & 5 & $100 \%$ & 8 & $100 \%$ & 8 & $100 \%$ & 3 & $100 \%$ & 30 & $100 \%$ \\
\hline
\end{tabular}

Fonte: dos autores.

Quanto ao tipo de pesquisa, destaca-se que a abordagem qualitativa (33\%), seguidas de quantitativa (3\%), quali-quanti (3\%) e 60\% que não declaram o tipo. Esses dados indicam que os autores buscaram estudar o tema de forma mais exploratória que são possíveis com os típicos de estudos qualitativos.

Observa-se que $77 \%$ dos trabalhos detalharam a metodologia. Apesar dessa maioria expressiva, observa-se ainda que estes detalhamentos focam-se no delineamento da pesquisa, observando-se poucos enquadramentos metodológicos em relação a paradigma, tipo de pesquisa, estratégia de pesquisa, métodos e análise de dados.

\subsection{Citações}

Um total de 704 referências bibliográficas e 946 citações foram observadas nos trabalhos. A composição desse referencial bibliográfico foi de $49,53 \%$ artigos de revista, $14,09 \%$ de livros, $14,20 \%$ de artigos de congressos e $22,18 \%$ de outros (incluindo teses, dissertações, boletins técnicos, dentre outros).

Os 10 autores mais citados são apresentados na Tabela 7.

Tabela 7 - 10 autores mais citados

\begin{tabular}{ccccc}
\hline \multirow{2}{*}{$\mathbf{N}^{\mathbf{0}}$} & Autor & Nacionalidade & \multicolumn{2}{c}{ Citado como autor } \\
\cline { 4 - 5 } & & EUA & 58 & 16 \\
\hline 1 & Glenn Ballard & Ro-autor \\
2 & Robin Cooper & Reino Unido & 39 & 3
\end{tabular}


Tabela 7 - 10 autores mais citados

\begin{tabular}{clccc}
\hline \multirow{2}{*}{$\mathbf{N}^{\mathbf{0}}$} & \multicolumn{1}{c}{ Autor } & Nacionalidade & \multicolumn{2}{c}{ Citado como autor } \\
\cline { 4 - 5 } & & & Principal & Co-autor \\
\hline 3 & Ana Mitsuko Jacomit & Brasil & 23 & 4 \\
4 & Ari Pennanen & Filândia & 19 & 0 \\
5 & Shahid Ansari & Paquistão & 16 & 1 \\
6 & Davide Nicolini & Itália & 13 & 0 \\
7 & Ariovaldo Dênis Granja & Brasil & 11 & 37 \\
8 & Yutaka Kato & Japão & 10 & 0 \\
9 & Doris C. C. K. Kowaltowsk & Alemanha & 10 & 3 \\
10 & Davood Askarany & Nova Zelândia & 9 & 1 \\
\hline
\end{tabular}

Fonte: dos autores.

Com relação aos congressos mais citados, observou-se um total de 36, dos quais os 5 merecem destaque conforme a Tabela 8 .

Tabela 8 - Congressos mais citados

\begin{tabular}{ccc}
\hline Congresso & Citações & \% do total \\
\hline IGLC & 73 & $53 \%$ \\
Joint CIB Intenational & 9 & $7 \%$ \\
Symposium & 7 & $5 \%$ \\
SIBRAGEC & 5 & $4 \%$ \\
ENTAC & 4 & $3 \%$ \\
\hline Construction Research Congress & 98 & $72 \%$ \\
\hline$\sum 5$ primeiros & 39 & $28 \%$ \\
\hline$\sum$ demais & $\mathbf{1 3 7}$ & $\mathbf{1 0 0 \%}$ \\
\hline Total &
\end{tabular}

Fonte: dos autores.

Com relação aos periódicos, observou-se um total de 197, dos quais os 10 mais citados representam quase $40 \%$ das citações, conforme a Tabela 9. 
Tabela 9 - 10 Periódicos mais citados nas referências bibliográficas

\begin{tabular}{ccc}
\hline Periódico & Citações & \% do total \\
\hline Ambiente Construído & 38 & $8,41 \%$ \\
International Journal of Production Economics & 30 & $6,64 \%$ \\
Journal of Cost Management & 20 & $4,42 \%$ \\
Management Accounting Research & 18 & $3,98 \%$ \\
Construction Management and Economics & 16 & $3,54 \%$ \\
Lean Construction Journal & 15 & $3,32 \%$ \\
British Journal of Management & 12 & $2,65 \%$ \\
International Journal of Project Management & 12 & $2,65 \%$ \\
Lean construction Journal & 10 & $2,21 \%$ \\
Journal of Construction Engineering and management & 9 & $1,99 \%$ \\
\hline$\sum 10$ primeiros & 180 & $39,82 \%$ \\
\hline$\sum$ demais & 272 & $56 \%$ \\
\hline Total & $\mathbf{4 5 2}$ & $\mathbf{1 0 0 \%}$ \\
\hline
\end{tabular}

Fonte: dos autores.

\section{CONSIDERAÇÕES FINAIS}

O presente artigo apresentou uma caracterização geral sobre a produção científica em target costing na construção civil durante os anos de 2009 a 2013 que resultou em uma amostra final de 30 publicações para o período.

Observou-se uma evidente polarização entre artigos de congresso $(63,33 \%)$ e artigos de revista $(26,27 \%)$. Liderando o ranking de fontes de publicação em custeio-meta encontra-se a conferência do International Group for Lean Construction (IGLC) com 36,67\% dos trabalhos (11 publicações) e os encontros da ANTAC (ENTAC e SIBRAGEC, com 5 publicações)

Os principais temas observados nas publicações relacionaram-se com gestão do processo de projetos $(27 \%)$, estimativas de custos $(23 \%)$, desenvolvimento de produtos (14\%) e habitação de interesse social (14\%), mapeamento da literatura (10\%), observando-se a diversidade de aplicações com que o tema vem sendo tratado.

Considerando o número de publicações e fator de ponderação foi possível observar os principais autores do tema: Ballard, Granja, Jacomit, Ruiz e Penanen. Quanto à titulação acadêmica destes autores constatou-se que $47 \%$ das pesquisas possuem doutores em suas equipes de pesquisa.

Destaca-se ainda a surpreendente colaboração Unicamp como instituição com maior colaboração nas publicações examinadas e que, juntamente com a Universidade da Califórnia-Berkeley são as únicas instituições que têm publicações em todos os anos examinados. Pode-se destacar, também, um maior número de publicações ligadas a instituições brasileiras com $36,37 \%$ seguidas de $31,82 \%$ por instituições Norte Americanas.

Observou-se que os trabalhos com estudo empírico predominaram com $83 \%$ do total. Destaca-se que a abordagem qualitativa obteve 33\%, seguida de 3\% quantitativa e $3 \%$ 
quali-quanti. Conclui-se que o estudo indica uma transição de estudos teóricos, embora ainda de forma incipiente, para estudos empíricos advindos de aplicações desta estratégia na construção civil remetendo a proposição inicial de um crescente avanço e maturação da utilização do target costing na construção civil.

\section{AGRADECIMENTOS}

A CAPES, pelo apoio recebido.

\section{REFERÊNCIAS BIBLIOGRÁFICAS}

CAMPOS, I. B; DIAS JÚNIOR, J. G; CARVALHO, A. B. VASCONCELOS, I. A de; BARROS NETO, J. P. Análise da produção científica sobre Lean Construction x Green Building no período de 2007 a 2011. In: XIV ENTAC - Encontro Nacional de Tecnologia do Ambiente Construído. Anais... Juiz de For a: 2012. pp. 3008-3016. CD-ROM.

BALLARD, G. Target Value Design: Current Benchmark (1.0). Jounal of Lean Construction, Arlington, USA, 2011, pp. 79-84, 2011.

COLLIS, J; HUSSEY, R. Pesquisa em administração: um guia prático para alunos de graduação e pós-graduação. 2 ed. Poro Alegre: Bookman, 2005.

FAPESP. Indicadores FAPESP de Ciência, Tecnologia e Inovação disponível em: http://www.fapesp.br/indicadores/boletim3.pdf Acesso em: dezembro de 2013.

GRANJA, A. D; JACOMIT, A. M; GUADANHIM, S. J; HIROTA, E. H. O custeio-meta para o desenvolvimento de habitações de interesse social: diretrizes a partir da comparação de duas modalidades de provisão. Revista Ambiente Construído, Porto Alegre, v. v. 11, n. 1, p. p. 53:66, 2011.

KERN, A. P. Proposta de um modelo de planejamento e controle de custos de empreendimentos de construção. Tese (Doutorado em Engenharia Civil) - Programa de Pósgraduação em Engenharia Civil, Universidade Federal do Rio Grande do Sul (UFRGS), Porto Alegre. 2005.

LACERDA, R. T. de O; ENSSLIN, L; ENSSILIN, S. R. Uma análise bibliométrica da literatura sobre estratégia e avaliação de desempenho. Gest. Prod., São Carlos, v. 19, n. 1, p. 59-78, 2012

OLIVEIRA, M. C. Análise dos periódicos brasileiros de contabilidade. Revista contabilidade \& Finanças - USP, São Paulo, n. 29, p. 68-86, maio/ ago. 2002.

PITHAN, D. N; AZAMBUJA, M. M. B; FORMOSO, C. T. BARROS NETO, J. P; Caracterização da produção científica de áreas de conhecimento específicas: aplicação à gestão e economia da construção. Revista Ambiente Construído, Porto Alegre, v. 5, n. 3, p. 7-18, jul./set. 2005.

ROCHA, I; WIENHAGE, P; SCARPIN, J. E. Investigação da produção científica relacionada ao Custeio-meta e Cuteio Kaizen no período de 2002 a 2009. ConTexto, Porto Alegre, v. 10, n. 18 , p. $75-86,2^{\circ}$ semestre 2010

RIBEIRO, C. K; PINHEIRO, L. V; de OLIVEIRA, E. da C. P. Construção de um modelosíntese para análise de periódicos científicos. In VIII ENANCIB - Encontro Nacional de Pesquisa em Ciência da Informação. 28 a 31 de outubro de 2007. Salvador, Bahia.

RICHARDSON, R. J. Pesquisa Social: Métodos e Técnicas. $3^{\text {a }}$ ed. São Paulo: Atlas, 2011. 